\title{
Original
}

\section{First J apanese Patient Treated with Parathyroid Hormone Peptide Immunization for Refractory Hypercalcemia Caused by Metastatic Parathyroid Carcinoma}

\author{
ICHIRO HORIE ${ }^{1)}$, TAKAO ANDO ${ }^{1)}$, NAOKO INOKUCHI ${ }^{2)}$, YUMI MIHARA ${ }^{3)}$, SHIRO MIURA ${ }^{3)}$, MiSA IMAIZUMI ${ }^{1)}$, \\ TOSHIRo USA ${ }^{1)}$, NAOE KINOSHITA ${ }^{4)}$, ICHIRO SEKINE ${ }^{3)}$, SHIMERU KAMIHARA ${ }^{2)}$ AND KATSUMI EGUCHI ${ }^{1)}$ \\ ${ }^{1)}$ First Department of Medicine, Nagasaki University School of Medicine, Nagasaki 852-8501, Japan \\ ${ }^{2)}$ Department of Laboratory Medicine, Nagasaki University School of Medicine, Nagasaki 852-8501, Japan \\ ${ }^{3)}$ Division of Tumor and Diagnostic Pathology, Nagasaki University School of Medicine, Nagasaki 852-8501, Japan \\ ${ }^{4)}$ Department of Pathology, Nagasaki University School of Medicine, Nagasaki 852-8501, Japan
}

\begin{abstract}
Patients with unresectable parathyroid carcinoma develop severe hypercalcemia, bone fractures and renal failure, and become unresponsive to conventional treatments. It has been shown that successful induction of anti-parathyroid hormone (PTH) antibodies, using PTH peptide fragments for immunisation, normalized serum levels of calcium as well as improved clinical symptoms. Here, we report our experience of PTH immunization in a Japanese female suffering from refractory hypercalcemia and renal failure caused by unresectable metastatic parathyroid carcinoma. Upon immunization, there were apparent clinical responses including reduction of serum levels of Ca along with anti-PTH antibodies induction. Therefore, we concluded that PTH immunization was an effective treatment against hypercalcemia caused by metastatic parathyroid carcinomas that are unresponsive to conventional treatments.
\end{abstract}

Key words: PTH, Parathyroid, Carcinoma, Metastasis, Immunization

PARATHYROID CARCINOMA is rare cause (less than 1\%) of primary hyperparathyroidism [1]. Typically, higher serum levels of parathyroid hormone (PTH) are seen in parathyroid carcinoma (3 to 10 times, or even higher, the upper limit of the reference range) than in parathyroid adenoma and hyperplasia and are associated with extreme hypercalcemia $(>14 \mathrm{mg} / \mathrm{dL})[1,2]$. There may be associated severe bone pain and ossteitis fibrosa cystica [1]. Parathyroid carcinoma is an indolent tumor with low malignant potential and, in general, has a tendency to recur locally in the operative site or spread into contiguous tissues in the neck. Distant metastases are a late phenomenon with lymphatic or hematogenous spread to cervical lymph nodes and lungs and less commonly to the liv-

Received Sep. 24, 2009; Accepted Dec. 8, 2009 as K09E-283 Released online in J-STAGE as advance publication Jan. 6, 2010

Correspondence to: Ichiro HORIE, First Department of Medicine, Nagasaki University School of Medicine, 1-7-1 Sakamoto, Nagasaki, Nagasaki 852-8501, Japan.

Email: ih1504@nagasaki-u.ac.jp er. The optimal treatment for parathyroid carcinoma is complete resection of the primary site, en bloc, including the surrounding tissues.

When metastatic parathyroid tumors are found, surgical resection of the metastatic tumors is the optimal treatment. This is because parathyroid carcinoma is refractory to radiation and cytotoxic reagents [1]. Medical management of hypercalcemia by infusing large amounts of fluid, along with bisphosphonates and perhaps calcitonin is briefly effective but, hypercalcemia eventually becomes uncontrolable. Recently, cinacalcet, a calcimimetic agent [3], has been used effectively to reduce hypercalcemia in patients with primary hyperparathyroidism [4] and two thirds of patients with parathyroid carcinoma also respond [5]. However, this treatment was not available in Japan for our patient in 2007, and, moreover, long-term efficacy has not been well established and calcimimetics do not inhibit proliferation of parathyroid cancer cells.

It was recently shown that immunization with PTH peptides was effective in controlling hypercalcemia 


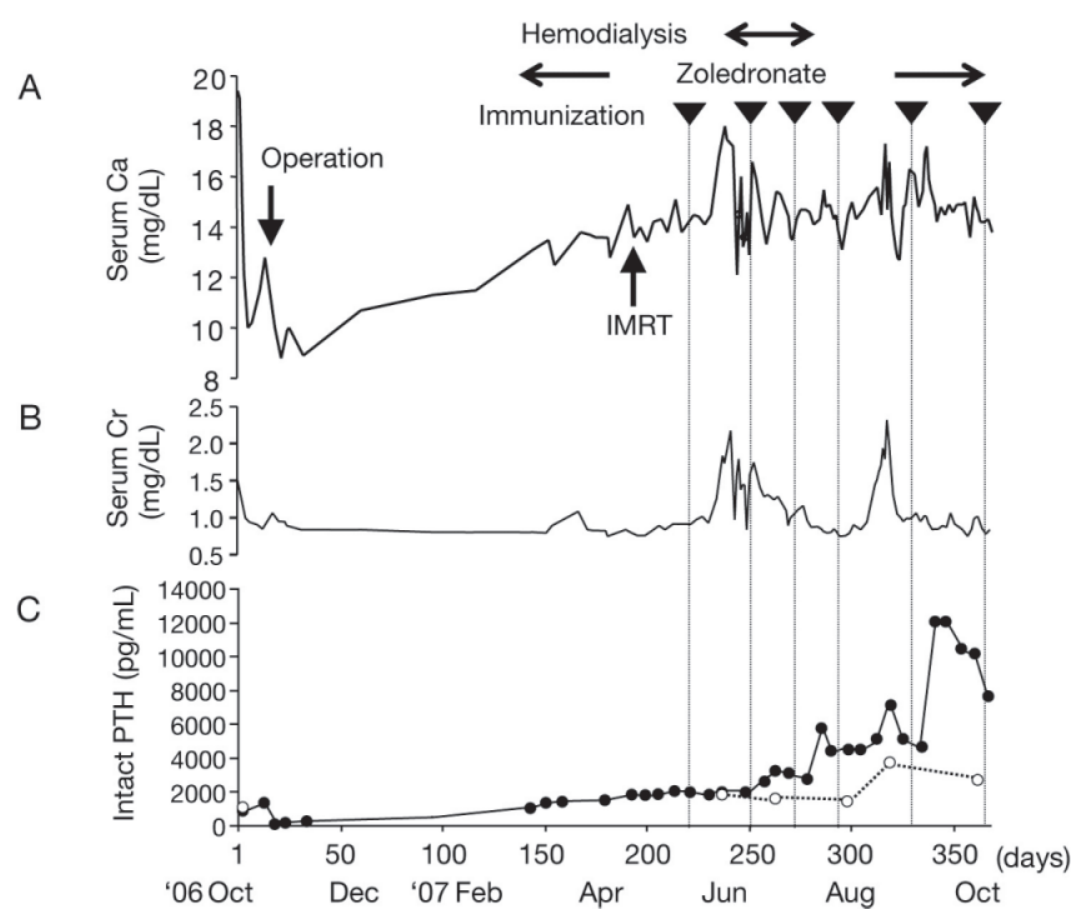

Fig. 1. Clinical course of the patient with parathyroid carcinoma treated with PTH immunization.

Serum levels of Ca (A), Cr (B), and intact PTH with (white dots) or without (black dots) PEG precipitation (C). Days after the first admission are indicated in the $\mathrm{X}$ axis. Note that the progressive increase in serum levels of $\mathrm{Ca}$ was stabilized after PTH immunization was initiated as indicated by black inversed triangles in the top of the panel (A), and also that dramatic reduction of PTH after PEG precipitation is apparent only after PTH immunization (C).

for more than 72 months in a patient with unresectable parathyroid carcinoma $[6,7]$ and caused resolution of metastatic tumor deposits [7]. This treatment is dependent on a strong host immune response against a limited set of PTH peptides immunogens and all patients were Caucasian [6-8]. Here we attempted PTH immunization in a Japanese patient suffering from uncontrolled hypercalcemia caused by unresectable parathyroid carcinoma.

\section{Patient}

A 34 year-old Japanese female was referred to our hospital because of markedly elevated serum levels of Ca (19.4 mg/dL; reference range: 9.0-10.6 mg/dL) in October 2006. Serum levels of intact PTH were elevated at $1148 \mathrm{pg} / \mathrm{mL}$; (reference range: 10.3-65.9 pg/mL; Eclusis PTH, Roche Diagnostic K.K., Tokyo, Japan) while serum levels of PTH-related peptide and angiotensin-converting enzyme were normal. Neck ultrasound and CT clearly identified two hypoechoic and low density nodules behind the right lobe of the thyroid which were also abnormal by ${ }^{99 m}$ Tc-methoxyisobutyl-isonitrile scintigraphy. Subsequently, the enlarged parathyroid glands were removed with the surrounding thyroid, en bloc, and a pathological diagnosis of parathyroid carcinoma was made with tumor invasion of the thyroid gland and veins. Postoperatively, serum levels of Ca normalized to below $10.0 \mathrm{mg} / \mathrm{dL}$. However, intact PTH remained higher than 200 pg/ $\mathrm{mL}$, indicating residual parathyroid tissue. However, we were not able to identify such lesions.

Over the following two months, there was a progressive increase in serum levels of Ca (Fig 1A) and intact PTH. At subsequent readmission the patient underwent a FDG-PET scan of the upper neck that identified a bone destructive tumor in the dental process (dens) in the $\mathrm{C} 2$ vertebra which was confirmed by CT and MRI. Since this was not surgically accessible, it was targeted with 50 Gray of intensity modulated radiation therapy (IMRT) with a peripheral margin of $2 \mathrm{~mm}$ receiving 45 Gray.

However, beneficial effects over the following month were not apparent. Serum levels of Ca were 
maintained at less than $15 \mathrm{mg} / \mathrm{dL}$ with a large amount of a fluid infusion combined with weekly zoledronate. After obtaining written informed consent from the patient and her family, and approval from the Nagasaki University Hospital ethic committee, we commenced PTH peptide immunotherapy.

\section{PTH peptides}

Immunogenic preparations of PTH peptides were generated as described previously $[6,7]$. Briefly, peptide sequences of human PTH1-34 (bioactive aminoterminal sequence), PTH33-52, and PTH 51-84 were synthesized. Single amino-acid modifications were made at position two of each peptide, and separately at position three for PTH1-34, in an attempt to improve antigenicity. Antigenicity was further increased by constructing octomeric, multi-antigenic peptides connected to a lysine core (Alta Biosciences, University of Birmingham, Birmingham, UK). Immunogens were dialyzed against sterile saline and combined in a proportion of 40:60 with complete Freund's adjuvant.

\section{Immunization procedure and detection of anti-PTH antibodies}

In each immunization, PTH peptides were given intradermally targeting the cervical, axillary, anterior thoracic, and inguinal lymph nodes. In the subsequent immunization, the alternative side of the body was used for immunization every 4-5 weeks. In the first three immunizations, only human PTH1-34 peptides were used. From the third immunization, we included not only PTH1-34 peptides but also PTH33-52 and PTH51-84 peptides as immunogens. Sera were collected before and two weeks after each immunization and anti-PTH antibodies were studied as previously described [6, 7].

\section{Study of free and antibody-bound PTH by polyethyl- ene glycol precipitation}

Polyethylene glycol precipitation (PEG) of sera was performed as previously described [9]. Briefly, sera were mixed with an equal volume of 25\% PEG6000 and the supernatants obtained by centrifuging were assessed for serum levels of intact PTH.

\section{Histological detection of PTH and Ki67}

Deparaffinized tissue sections obtained at surgical operation and autopsy were stained with anti-PTH mouse monoclonal antibodies (1:200) (Novocastra,

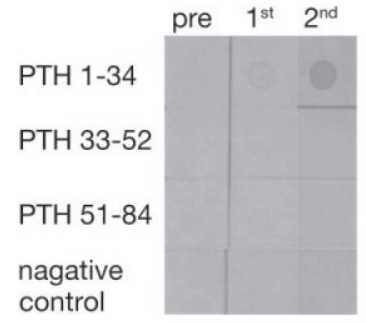

(A)

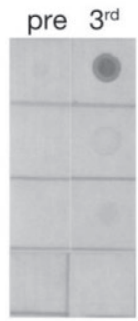

(B)

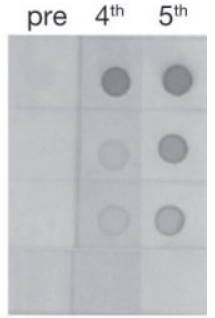

(C)
Fig. 2. Anti-PTH antibodies detected by dot blots.

Recognition of the patient's sera to three PTH peptides studied by dot blots analysis. Sera collected two weeks after the first and second (A), third (B), and fourth and fifth immunization $(C)$ were studied. Sera collected prior to the immunization (pre) and the unrelated peptide were also included as negative antibody control and as negative antigen control, respectively.

Newcastle, UK) or Ki 67 antibodies (1:100) (Dako, Japan) using routine methods [10].

\section{Results}

After the first immunization, using $\mathrm{N}$ terminus of the PTH (PTH1-34), there was a pyrexia of over $39^{\circ} \mathrm{C}$ for 3 days, then skin ulcerations developed at all seven immunization sites. Anti-PTH antibodies were weakly detectable in sera taken two weeks later (Fig 2A). However, while awaiting second immunization, the patient developed a Ca crisis (serum Ca $>18 \mathrm{mg} / \mathrm{dL}$ ) and the serum levels of creatinine increased from 0.9 to $2.2 \mathrm{mg} / \mathrm{dL}$ within a week (Fig 1B). The patient became oliguric and we began hemodialysis. However, serum Ca concentrations never decreased below 16 $\mathrm{mg} / \mathrm{dL}$.

After the second immunization with PTH 1-34, serum levels of intact PTH increased from 2,036 to 3,627 $\mathrm{pg} / \mathrm{mL}$ while serum levels of Ca decrease to $14 \mathrm{mg} /$ dL. This suggested the presence of immunoglobulin/ PTH immune complexes, a supposition that was supported by rising titres of antibodies to PTH1-34 (Fig 2B) observed using dot-blot analysis. It was decided to stop hemodialysis.

Having inducing a good immune response to PTH134, $\mathrm{N}$ terminus as well as middle (PTH33-53) and C terminus (PTH52-84) immunogens were used from the third to fifth immunization. The reason of not including middle or $\mathrm{C}$ terminus peptides in the first and second immunization was due to a concern of a potential activation of PTH receptor by IgG-bound PTH, which 
seems to have longer half-life than PTH itself, without inducing antibodies to PTH N terminus. Serum levels of intact PTH rose further to $\sim 6,000, \sim 7,500$, and finally $\sim 12,000 \mathrm{pg} / \mathrm{mL}$ after the third, fourth, and fifth immunization, respectively. Dot-blot analysis showed progressive increases in antibody titers to all the PTH fragments (Fig 2B and C). Correspondingly, serum Ca decreased below $14 \mathrm{mg} / \mathrm{dL}$ within a week after each immunization. Unfortunately, such beneficial effects were transient and serum levels of Ca gradually increased to over $15 \mathrm{mg} / \mathrm{dL}$ within 3 weeks of the injections. Therefore, immunization was repeated every four weeks.

Such transient therapeutic effects of the PTH immunization were not as evident in previously successfully treated patients [6-8]. Since the antibody titres were high, yet ineffective, we considered that parathyroid carcinoma was either growing rapidly or had highly efficient PTH production. Subsequent whole body CT scans detected multiple metastases in the lungs and liver. There was an additional osteolytic lesion in the neck of the left femur (not shown), that was also considered to be metastatic tissue. These lesions had not been identified before the PTH immunization indicating a highly aggressive tumor. This offered an explanation for the poor clinical result inspite of effective immunization.

From the clinical picture it was obvious that the patient was terminally ill, after extensive discussions with family members about the advantages and disadvantages of continuing immunization in the current clinical status, 6th immunization was performed. However, a high-output heart failure developed leading to hypotensive shock and death.

At postmortem, we identified a small $(7 \mathrm{~mm})$ subpleural nodule in the right lung and multiple masses in the liver (5-20 $\mathrm{mm}$ in the diameter) and a metastasis in the dens of the second vertebra. Microscopic studies showed cell proliferation with palisading, rosetteformation and honeycomb structures characteristic of endocrine tumors (Fig 3A), and cells stained positively with anti-PTH antibody (Fig 3B). Proliferating cells, as studied by Ki-67 labeling indices, were $2.1 \%$ in the primary tumor (Fig 3C), $0.4 \%$ in the metastatic lung tumor (Fig 3D) and $0.8 \%$ in the metastatic liver tumor (not shown). There was no apparent tumor necrosis or fibrosis in the metastatic lesion in the second cervical vertebra (not shown) where IMRT had been targeted. Her heart displayed severe concentric hyper-

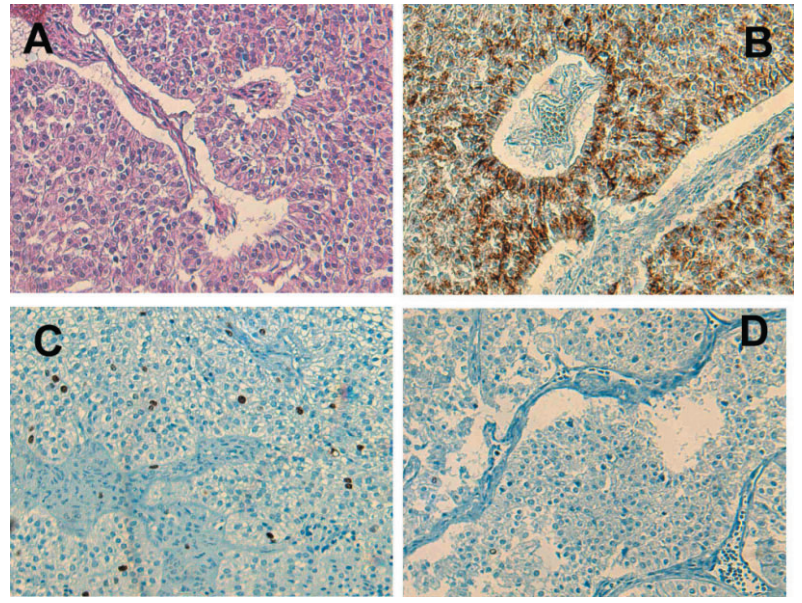

Fig. 3. Microscopic findings of the postmortem studies.

The metastatic lung tumors showing a uniform chief cell proliferation with sheet-like arrangement with nuclear palisading and rosette-like structure (A). Parathyroid cancer cells positively stained with anti-PTH antibody (B). Proliferating cells in the primary tumor (C) and metastatic liver tumor (D) stained with Ki-67 antibody. Hematoxyline and eosin staining (A) and immunostaining for PTH (B) and Ki-67 (C and D). Magnifications are $\mathrm{x} 400$.

trophy. There was severe pulmonary edema accounting for death by heart failure.

Having shown that PTH immunization was successful in this patient, we wondered how serum levels of Ca fell down in the presence of elevating serum levels of intact PTH. Since the immunization induced antiPTH antibodies, we decided to measure free and antibody-unbound PTH in the sera. By utilizing PEG precipitation, it was clear that the free PTH levels were much less after immunizations (Fig 1C).

\section{Discussion}

PTH immunization was originally attempted in a patient with uncontrolled hypercalcemia caused by metastatic parathyroid carcinoma[6]. Subsequently, several patients with unresectable parathyroid carcinoma have been treated [6-8]. Most of the enrolled patients showed positive immune response to PTH peptides, as determined by dot blots, showing favorable clinical responses including reduced concentrations of serum Ca. Clinical responses in responders normally occurred after the first or second immunization. In addition to lowering serum levels of Ca, tumor shrinkage was seen in a patient upon immunization [7]. Adverse 
events of the treatment so far observed were not serious, such as high fever and skin ulcerations at the sites of immunization, which are also indicators of good immune response of the host. Here we showed that a Japanese with parathyroid carcinoma responded to the PTH immunization as indicated by a progressive increase in antibody titers after each immunization and these antibodies neutralized PTH bioactivity as indicated by PEG precipitation. Reduced serum levels of Ca occurred despite higher PTH concentrations. Such beneficial effects were apparent not only biologically but also clinically.

Despite a positive immunological response, the parathyroid carcinoma in our patient showed aggressive tumor growth and rapid metastatic spread. It is known that first recurrences are typically detected at around 3 years after surgery but the interval may be much longer [1]. Even after palliative surgery of the recurrent parathyroid carcinoma, 10-year survivals of nearly $50 \%$ are reported [11]. It has been previously shown that nuclear atypia and higher mitosis were seen in those who died of parathyroid carcinoma in one retrospective study [12]. It has been reported from our country that prognostic factors of parathyroid carcinoma were loco-regional extension of the tumor, type of surgical procedure used in the initial operation, and high $(>5 \%)$ index of cell division [13]. Since the cell division indices in our patient were $<5 \%$, the explanation for the aggressive nature of the tumor is unclear.

It is known that parathyroid carcinoma is radio-re- sistant and it has been shown that radiation therapy was largely ineffective to control tumor growth and decrease hormone production $[14,15]$ with exceptional papers $[16,17]$. IMRT is to irradiate the target by using several radiation beams of varying intensity and is expected to improve dose homogeneity while sparing the surrounding important tissue nearby the target because of its way of irradiation [18]. However, as described, IMRT was not effective to metastatic parathyroid carcinoma in our patient.

In summary, we report our experience of PTH immunization performed on a Japanese patient with metastatic parathyroid carcinoma. Since our case was highly aggressive, there was minimal remission of hypercalcemia. However, PTH immunization effectively provoked a host immune response against PTH peptides and partly neutralized PTH produced from the tumor. Therefore, PTH immunization should be considered for patients with severe hypercalcemia caused by metastatic parathyroid carcinomas that are unresponsive to conventional treatments.

\section{Acknowledgements}

We thank Dr Arthur R. Bradwell, the Division of Immunity and Infection, Medical School University of Birmingham, Birmingham, United Kingdom, for his generosity to provide us PTH peptides and to examine serum anti-PTH antibodies by dot blots, as well as his critical suggestions to the manuscript.

\section{References}

1. Shane E (2001) Clinical review 122: Parathyroid carcinoma. J Clin Endocrinol Metab 86: 485-493.

2. Hakaim AG, Esselstyn CB, Jr. (1993) Parathyroid carcinoma: 50-year experience at The Cleveland Clinic Foundation. Cleve Clin J Med 60: 331-335.

3. Dong BJ (2005) Cinacalcet: An oral calcimimetic agent for the management of hyperparathyroidism. Clin Ther 27: 1725-1751.

4. Silverberg SJ, Bone HG, 3rd, Marriott TB, Locker FG, Thys-Jacobs S, Dziem G, Kaatz S, Sanguinetti EL, Bilezikian JP (1997) Short-term inhibition of parathyroid hormone secretion by a calcium-receptor agonist in patients with primary hyperparathyroidism. $N$ Engl $J$ Med 337: 1506-1510.

5. Silverberg SJ, Rubin MR, Faiman C, Peacock M, Shoback DM, Smallridge RC, Schwanauer LE, Olson
KA, Klassen P, Bilezikian JP (2007) Cinacalcet hydrochloride reduces the serum calcium concentration in inoperable parathyroid carcinoma. J Clin Endocrinol Metab 92: 3803-3808.

6. Bradwell AR, Harvey TC (1999) Control of hypercalcaemia of parathyroid carcinoma by immunisation. Lancet 353: 370-373.

7. Betea D, Bradwell AR, Harvey TC, Mead GP, Schmidt-Gayk H, Ghaye B, Daly AF, Beckers A (2004) Hormonal and biochemical normalization and tumor shrinkage induced by anti-parathyroid hormone immunotherapy in a patient with metastatic parathyroid carcinoma. J Clin Endocrinol Metab 89: 3413-3420.

8. Mead GP, Harvey T, Epstein M, Beckers A, Bradwell AR (2002) Hypercalcaemia of parathyroid carcinoma controlled by immunotherapy. Program of the $84^{\text {th }}$ 
Annual Meeting of The Endocrine Society, OR21-23 (Abstract)

9. Ando T, Yasui J, Inokuchi N, Usa T, Ashizawa K, Kamihara S, Eguchi K (2007) Non-specific activities against ruthenium crosslinker as a new cause of assay interference in an electrochemilluminescent immunoassay. Intern Med 46: 1225-1229.

10. Nakazato K, Nakajima K, Kusakabe T, Suzuki K, Nagamine T (2008) Immunohistochemical staining with newly developed metallothionein fragment antibodies against NH2-terminal, middle-regional and $\mathrm{COOH}$ terminal peptides in rabbits. Pathol Int 58: 765-770.

11. Hundahl SA, Fleming ID, Fremgen AM, Menck HR (1999) Two hundred eighty-six cases of parathyroid carcinoma treated in the U.S. between 1985-1995: a National Cancer Data Base Report. The American College of Surgeons Commission on Cancer and the American Cancer Society. Cancer 86: 538-544.

12. Sandelin K, Tullgren O, Farnebo LO (1994) Clinical course of metastatic parathyroid cancer. World J Surg 18: 594-598.

13. Iihara M, Okamoto T, Suzuki R, Kawamata A, Nishikawa T, Kobayashi M, Obara T (2007) Functional parathyroid carcinoma: Long-term treatment outcome and risk factor analysis. Surgery 142: 936-943.

14. Cohn K, Silverman M, Corrado J, Sedgewick C (1985) Parathyroid carcinoma: the Lahey Clinic experience. Surgery 98: 1095-1100.

15. Schantz A, Castleman B (1973) Parathyroid carcinoma. A study of 70 cases. Cancer 31: 600-605.

16. Chow E, Tsang RW, Brierley JD, Filice S (1998) Parathyroid carcinoma - the Princess Margaret Hospital experience. Int J Radiat Oncol Biol Phys 41: 569-572.

17. Levin KE, Galante M, Clark OH (1987) Parathyroid carcinoma versus parathyroid adenoma in patients with profound hypercalcemia. Surgery 101: 649-660.

18. Spoelstra FO, Senan S (2008) Novel tools in radiotherapy. Ann Oncol 19 Suppl 7: vii294-299. 